Dem Bundeswirtschaftsministerium, Bonn (Forschungsvorhaben 822), der A r b eits. gemeinschaft industrieller Forschungsvereinigungen, Köln, dem Landesam t für Forschung beim Ministerpräsidenten des Landes Nordrhein-Westfalen, Düsseldorf, dem Forschungskuratorium Gesam t - textil, Frankfurt, und der De u t s chen F or s chungsgemeinschaft, Bad Godesberg, danken wir für finanzielle Unterstützung der vorliegenden Arbeit. Dem Verband der chemischen Industrie und seinen Mitgliedsfirmen danken wir für die Überlassung von Chemikalien im Rahmen von Hochschullieferungen.

\title{
Synthese einer teilgeschützten A-Kette des Schafinsulins ****
}

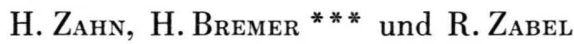 \\ Deutsches Wollforschungsinstitut an der Rheinisch-Westfälischen Technischen Hochschule Aachen
}

(Z. Naturforschg. 20 b, 653-660 [1965] ; eingegangen am 3. Mai 1965)

\begin{abstract}
Die A-Kette des Schafinsulins wurde als teilgeschütztes Derivat Carbobenzoxy-glycyl-L-isoleucyl-Lvalyl-L-glutamyl-L-glutaminyl-S-benzyl-L-cysteinyl-S-benzyl-L-cysteinyl-L-alanyl-glycyl-L-valyl- $S$ - benzylL-cysteinyl-L-seryl-L-leucyl-L-tyrosyl-L-glutaminyl-L-leucyl-L-glutamyl-L-asparaginyl-L-tyrosyl- $S$-benzyl-Lcysteinyl-L-asparagin synthetisiert und in dieser Form durch Gelfiltration über Sephadex G 25 in wäßriger Lösung bei $p_{\mathrm{H}} 8,5-8,7$ gereinigt. Das synthetische Material entspricht dem natürlichen in biologischen und chemischen Testen.
\end{abstract}

1963 wurde in dieser Zeitschrift über eine Synthese der A- und B-Kette des Schafinsulins und deren Kombination zu insulinaktivem Material in einer Kurzmitteilung berichtet ${ }^{1}$. Die Aminosäuresequenz des Schafinsulins ${ }^{2}$ (Abb. 1) wurde deshalb für die Synthese gewählt, weil sie im Gegensatz zu den Sequenzen anderer Insuline in der Position 9 der A-Kette einen Glycinrest aufweist. Dies ermöglicht die Synthese der aus 21 Aminosäuren bestehenden A-Kette aus den Fragmenten A $1-9$ und A 10 bis 21 nach jeder Methode der Carboxylgruppen-
Aktivierung, ohne daß man Racemisierung befürchten müßte.

Eine ähnliche Synthese der A-Kette des Schafinsulins wurde von Katsoyannis und Mitarbb. publiziert $^{3}$. Unterschiede zu unserer Synthese bestehen in der Wahl einiger Schutzgruppen und Kondensationsmethoden. $\mathrm{W}_{\text {ANG }}$ et al. ${ }^{4}$ synthetisierten unlängst auch die A-Kette des Rinderinsulins auf vergleichbarem Wege.

Für die Synthese benötigten wir zwei Teilpeptide (vgl. Schema 1), die durch Fragmentkondensation

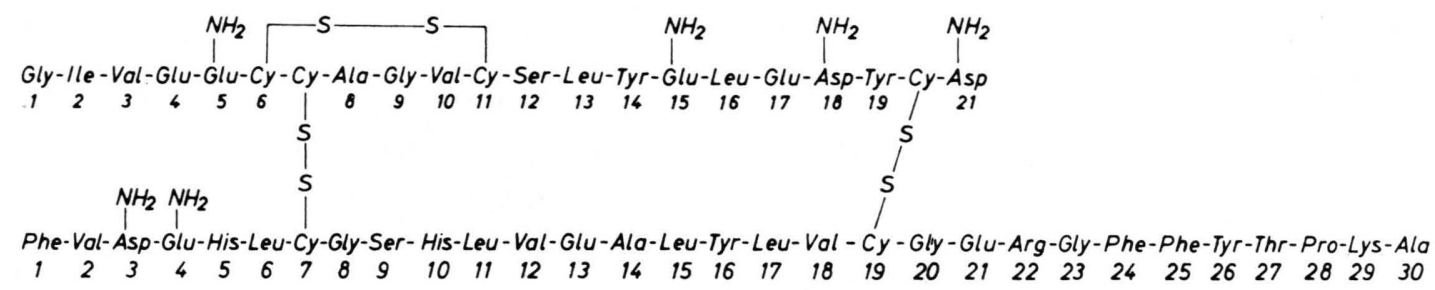

Abb. 1. Aminosäureformel des Schafinsulins.

* 47. Mitt. über Peptide. 46. Mitt.: R. Z Z $\mathrm{ABEL}$ u. H. $\mathrm{Z}_{\mathrm{AHN}}$, voranstehend.

** Abkürzungen nach E. Brand u. J. T. Edsall, Ann. Rev. Biochm. 16, 224 [1947] sowie Peptides - Proceedings of the 5th European Symposium Oxford 1962, Pergamon Press 1963.

*** Teil der Diss. H. Bremer, Rheinisch-Westfälische Technische Hochschule Aachen, 1964.

1 J. Meienhofer, E. Schnabel, H. Bremer, O. Brinkhoff, R. Zabel, W. Sroka, H. Klostermeyer, D. Brandenburg, T. Okuda u. H. Zahn, Z. Naturforschg. 18 b, 1120 [1963].
2 H. Brown, F. Sanger u. R. Kitai, Biochem. J. 60, 556 [1955].

3 P. G. Katsoyannis, A. Tometsko u. K. Funuda, J. Amer. chem. Soc. 85, 2863 [1963].

4 Y. Wang, J.-z. Hsu, W.-c. Chang, L.-L. Cheng, C.-y. Hsing, A.-н. Chi, T.-p. Loh, C.-н. Li, P.-т. SHI u. Y.-н. YieH, Sci. Sinica [Peking] 13, 2030 [1964].

5 R. A. Borssonnas, Helv. chim. Acta 34, 874 [1951]; J. R. VAughan, J. Amer. chem. Soc. 73, 3547 [1951]; TH. W land u. H. Bernhard, Liebigs Ann. Chem. 572, 190 [1951]. 

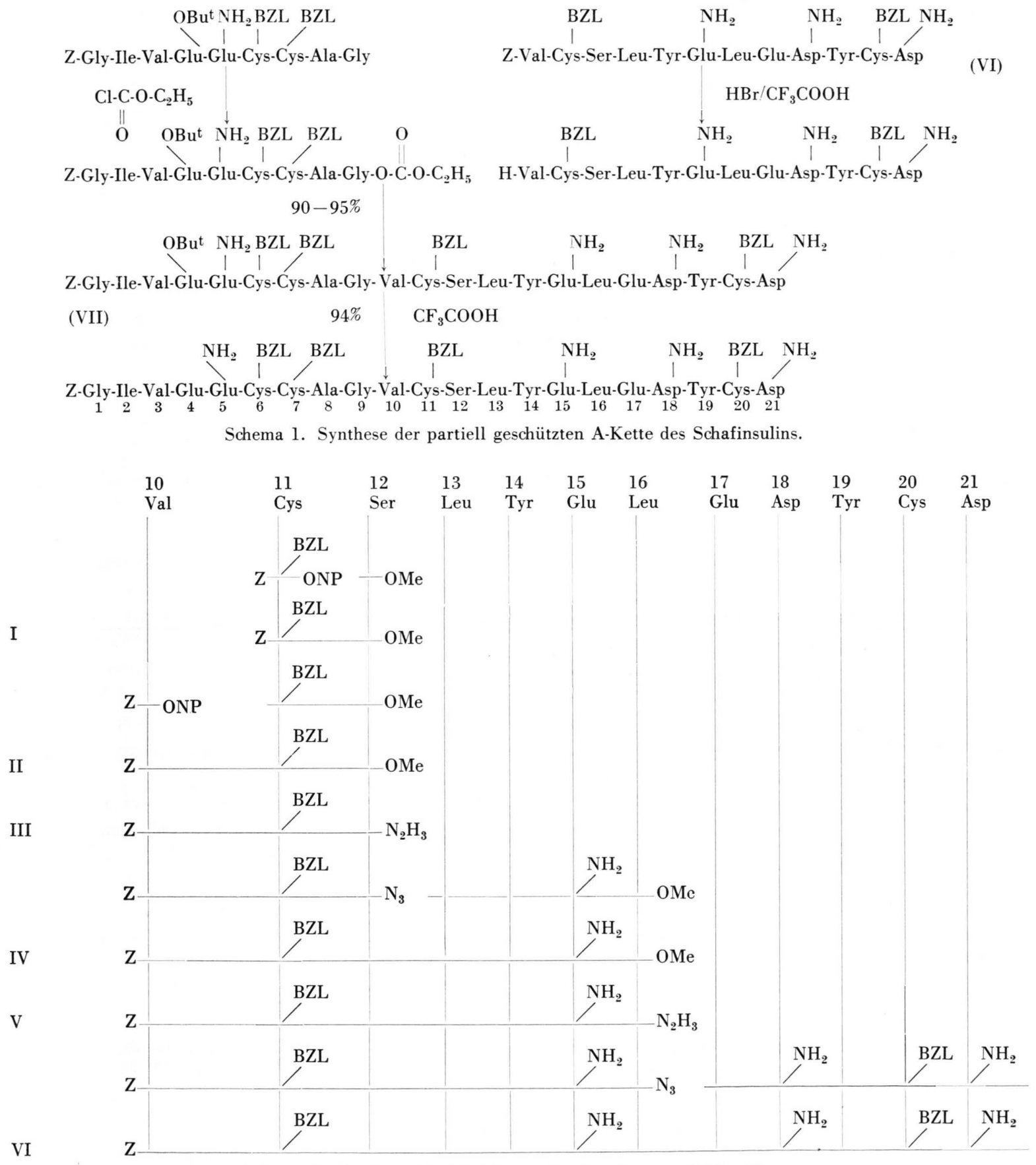

Schema 2. Synthese des Dodekapeptids der Sequenz A 10-21.

aus insgesamt fünf kleineren Peptiden aufgebaut wurden.

Die Synthese der Teilsequenzen A $1-4^{6}$, A 5 bis $9^{6}$ und A $1-9^{6}$ sowie A $17-21^{7}$ ist in den

${ }^{6}$ H. Zahn, H. Bremer, W. Sroka u. J. Meienhofer, Z. Natur forschg. 20 b, 646 [1965]. beiden voranstehenden Arbeiten beschrieben. Hier folgt die Beschreibung der Synthesen des Teilpeptides A 10-21 und des teilgeschützten Heneikosipeptides A $1-21$.

7 R. Z $\mathrm{ABEL}$ u. H. Z $\mathrm{AHN}$, Z. Naturforschg. 20 b, 650 [1965]. 
Synthese des Dodekapeptids A 10-21 (Schema 2)

Das Tripeptid der Sequenz A 10-12 wurde als Z-Val-Cys (BZL)-Ser-OMe * nach der Nitrophenylester-Methode dargestellt und mit Hydrazinhydrat in Dimethylformamid/Butanol-2 (1:1) in das Hydrazid Z-Val-Cys (BZL) -Ser- $\mathrm{N}_{2} \mathrm{H}_{3}$ * überführt. Die Gesamtausbeute über alle Stufen betrug $40 \%$ der Theorie. Die Darstellung des Dipeptids Z-Cys (BZL) Ser-OMe verlief ohne Nebenreaktionen in Dimethylformamid, worin die Kupplungskomponenten vollständig gelöst waren. In Äthylacetat oder Tetrahydrofuran ist das Serinmethylesterhydrochlorid dagegen nur wenig löslich und die Freisetzung des Esters erfolgt langsam. Für die Kondensation ist also ein Unterschuß Serinester verfügbar. Aus dem Reaktionsprodukt solcher Ansätze konnten bis 25\% eines Nebenproduktes isoliert werden, dessen Elementaranalyse mit der Formel

$$
[\mathrm{Z}-\mathrm{Cys}(\mathrm{BZL})]_{2}>\mathrm{Ser}-\mathrm{OMe}
$$

übereinstimmt.

Zur Darstellung des Heptapeptids mit der Sequenz A 10 - 16 wurde das Hydrazid durch Umsetzung mit Salpetriger Säure in das Azid Z-Val-Cys(BZL) -Ser- $\mathrm{N}_{3}$ überführt und dieses mit dem Tetrapeptid Leu-Tyr-Glu $\left(\mathrm{NH}_{2}\right)$-Leu-OMe ${ }^{*}$ gekuppelt. Die Kupplung verlief in Äthylacetat/Dimethylformamid mit 53\% Ausbeute. Der erhaltene Heptapeptidmethylester wurde durch Einwirkung von Hydrazinhydrat in Dimethylformamid in das Hydrazid Z-Val-Cys(BZL) -Ser-Leu-Tyr-Glu $\left(\mathrm{NH}_{2}\right)$-Leu- $\mathrm{N}_{2} \mathrm{H}_{3}$ umgewandelt.

Das gewünschte Dodekapeptid der Sequenz A 10 bis 21 wurde nun durch Kondensation des Heptapeptidazids A $10-16$ mit dem Pentapeptid-Derivat Glu-Asp $\left(\mathrm{NH}_{2}\right)-\mathrm{Tyr}-\mathrm{Cys}(\mathrm{BZL})-\operatorname{Asp}\left(\mathrm{NH}_{2}\right) * *$ in Dimethylformamid als Z-Val-Cys (BZL)-Ser-Leu-TyrGlu $\left(\mathrm{NH}_{2}\right)$ - Leu - Glu - Asp $\left(\mathrm{NH}_{2}\right)$ - Tyr - Cys (BZL) - Asp $\left(\mathrm{NH}_{2}\right)$ erhalten. Das Präparat ist nicht kristallisiert und wurde wiederholt mit Methanol ausgekocht, bis im Dünnschichtchromatogramm keine Nebenprodukte mehr erkennbar waren. Das Ergebnis der quant. Aminosäureanalyse ${ }^{8}$ eines 48-Stdn.-Hydrolysates bei $105^{\circ}$ in $6-n$. Salzsäure mit $10 \%$ Trifluoressigsäure-Zusatz als Lösungsvermittler ergab gute

* Diese Derivate sind unabhängig von uns auch von P. G. Katsoyannis und Mitarbeitern dargestellt worden, vgl. 1. c. $\left.{ }^{3}\right)$.

* Synthese des entsprechenden N-geschützten Peptids Z-LeuTyr-Glu $\left(\mathrm{NH}_{2}\right)$-Leu-OMe: l. c. ${ }^{3}$.
Übereinstimmung der erwarteten und gefundenen Aminosäureverhältnisse: Val $0,71(1,00)$, Ser 0,83 $(1,00)$, Leu 2,08 (2,00), Tyr 1,68 (2,00), Glu 2,06 $(2,00)$, Asp 2,00 (2,00); Cystein anwesend, aber nicht bestimmt (in Klammern theoretische Werte, Berechnungsbasis Asp). Im Hydrolysat wurde außer den vorhandenen Aminosäuren papierchromatographisch eine weitere Substanz ermittelt, bei der es sich vermutlich um Val-Cys (BZL) handelt. Die zu niedrigen Werte für Serin und Tyrosin sind auf Zersetzung während der Hydrolyse zurückzuführen.

Synthese des Heneikosipeptids A 1-21 (Schema 1)

Zur Synthese des Heneikosipeptids A $1-21$ wurde das Nonapeptid-Derivat Z-Gly-Ile-Val-Glu $\left(\mathrm{OBu}^{\mathrm{t}}\right)$ Glu- $\left(\mathrm{NH}_{2}\right)$-Cys (BZL) - Cys (BZL) -Ala-Gly in Hexamethylphosphorsäuretriamid mit Chlorameisensäureäthylester in das gemischte Anhydrid ${ }^{5}$ übergeführt und mit einer Lösung des decarbobenzoxylierten Dodekapeptids Val-Cys(BZL)-Ser-Leu-Tyr-Glu( $\left.\mathrm{NH}_{2}\right)$ Leu-Glu-Asp $\left(\mathrm{NH}_{2}\right)$-Tyr-Cys (BZL)-Asp $\left(\mathrm{NH}_{2}\right)$ in dem gleichen Lösungsmittel umgesetzt. Bei der ersten Synthese waren zwei Äquivalente des NonapeptidDerivates eingesetzt worden. Mit Wasser wurden
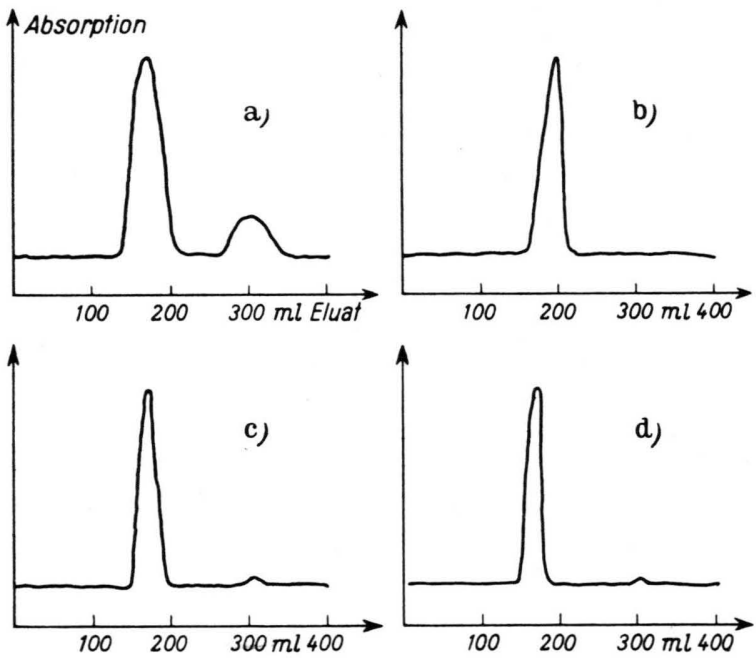

Abb. 2. Gelfiltration der teilgeschützten A-Kette (VII) in 0,1-m. TRIS-Lösung. a) 1. Ansatz (2 Äquivalente Nonapeptid). b) Zweiter Durchlauf von Peak I, $[a]_{\mathrm{D}}^{25}=-51^{\circ}$ c) 2. Ansatz (1 Äquivalent Nonapeptid) $[a]_{\mathrm{D}}^{25}=-48^{\circ}$. d) 2. Ansatz nach Auskochen mit Methanol $[a]_{\mathrm{D}}^{25}=-50,5^{\circ}$.

\footnotetext{
* Synthese des geschützten Peptids Z-Glu $\left(\mathrm{OBu}^{\mathrm{t}}\right)$-Asp $\left(\mathrm{NH}_{2}\right)$ Tyr-Cys (BZL)-Asp $\left(\mathrm{NH}_{2}\right)$ : l. c. ${ }^{7}$.

8 St. Moore, D. H. Spackman u. W. H. Stein, Analytic. Chem. 30, 1185 [1958].
} 
$30 \%$ mehr Produkt ausgefällt, als man an Heneikosipeptid erwartete. Das überschüssige NonapeptidDerivat war also mit ausgefallen und konnte weder durch Umfällen noch durch Auskochen mit Methanol abgetrennt werden.

Das Heneikosipeptid war aber nach der Abspaltung der tert.-Butylestergruppe als Anion wasserlöslich, damit ergab sich die Möglichkeit der Gelfiltration (Abb. 2) an Sephadex G 25 in TRIs-Puffer bei $p_{\mathrm{H}} 8,5-8,7$.

Bei den späteren Synthesen wurden äquivalente Mengen Nona- und Dodekapeptid eingesetzt. Die Gelfiltration lieferte nur noch $2-4 \%$ Nonapeptid, während das Heneikosipeptid-Derivat in einer Ausbeute von $90-95 \%$ anfiel.

Für das so gereinigte Heneikosipeptid-Derivat wurde eine befriedigende Elementaranalyse erhalten (vgl. experimenteller Teil). Die Ergebnisse der quantitativen Aminosäureanalyse sind in der folgenden Tabelle zusammengefaßt:

\begin{tabular}{|c|c|c|c|}
\hline \multirow{2}{*}{\multicolumn{2}{|c|}{$\begin{array}{l}\text { Berechnete } \\
\text { Molzahlen }\end{array}$}} & \multicolumn{2}{|c|}{ Gefunden nach Hydrolyse in } \\
\hline & & $\begin{array}{c}\text { 6-n. } \mathrm{HCl}+10 \% \text { Tri- } \\
\text { fluoressigsäure } \\
\text { nach } 72 \mathrm{Stdn} \text {. bei }\end{array}$ & $\begin{array}{l}\text { 13- } n \text {. HCl nach } \\
85 \mathrm{Stdn} \text {. bei }\end{array}$ \\
\hline Gly & 2,00 & 2,00 & Bezugswert \\
\hline Asp & 2,00 & 1,88 & \\
\hline Ser & 1,00 & 0,88 & \\
\hline Glu & 4,00 & 4,54 & \\
\hline Ala & 1,00 & 1,11 & \\
\hline Val & 2,00 & 1,25 & 2,00 \\
\hline Ile & 1,00 & 0,40 & 0,86 \\
\hline Leu & 2,00 & 2,26 & 2,26 \\
\hline Tyr & 2,00 & 1,58 & 0,20 \\
\hline Cys (BZL) & 4,00 & 3,23 & \\
\hline
\end{tabular}

Tab. 1. Quantitative Aminosäureanalyse des durch Gelfiltration gereinigten Heneikosipeptids.

Die Amidgruppen-Bestimmung an einem 17-Stdn.Hydrolysat bei $105^{\circ}$ in 13- $n$. HCl ergab 3,65 (berechnet 4,00) Amidgruppen pro Mol Peptid.

Dem hier beschriebenen Peptid-Derivat lag die von SANGER ${ }^{2}$ angegebene Formel der Insulin-A-Kette zugrunde. Es diente auf zweierlei Weise zum Beweis der durch Abbau ermittelten Struktur des nativen Materials:

A) Die synthetische Insulin-A-Kette lieferte bei der Kombination mit synthetischer ${ }^{1,9}$ und natürli-

${ }^{9}$ H. Z Zahn, J. Meienhofer u. O. Brinkhoff sowie E. F. Pfeiffer u. H. Ditschuneit u. C. Gloxhuber, Z. Naturforschg. 20 b, 666 [1965].

10 British Pharmacopoeia, pp. 816-819, The Pharmaceutical Press, London 1956. cher (s. exp. Teil) Insulin-B-Kette insulinaktive Präparate, deren Aktivitäten in der zu erwartenden Größenordnung lagen. Die Substanzen wurden nach drei verschiedenen Verfahren in drei Laboratorien getestet, die Ergebnisse (vgl. Tab. 1) sprechen zwar für die Richtigkeit der Formel von SANGER, stellen allein aber noch keinen Beweis dar, da vielleicht auch insulinähnliche Proteine dieselbe Aktivität besitzen (zur Aktivität der oxydierten A-Kette allein vgl. l. c. ${ }^{9}$ ).

\begin{tabular}{|c|c|c|c|}
\hline \multirow{2}{*}{ Präparat } & \multicolumn{3}{|c|}{$\%$ Insulinaktivität } \\
\hline & $\begin{array}{l}\text { Mauskrampf- } \\
\text { test }{ }^{10}\end{array}$ & $\begin{array}{c}\text { Glucose- } \\
\text { verbrauch }\end{array}$ & $\begin{array}{c}\text { Glucose- } \\
\text { oxydation }\end{array}$ \\
\hline \multirow{3}{*}{$\begin{array}{l}\text { Nat. } A+\text { nat.B } \\
\text { Synth. } A_{1}+ \\
\text { nat. B } \\
\text { Synth. } A_{2}+ \\
\text { nat. B }\end{array}$} & $1-2$ & $1-2$ & $1-2$ \\
\hline & $0,5-1,0$ & $0,2 *$ & 0,67 \\
\hline & 1,08 & $2-3$ & - \\
\hline
\end{tabular}

Tab. 2. Insulinaktivität halbsynthetischer Insuline; Bezugsstandard $=25$ I.E. $/ \mathrm{mg}$.

$A_{1}=$ erster Ansatz, mit überschüssigem Nonapeptid dargestellt. $A_{2}=$ zweiter Ansatz aus äquivalenten Mengen Nonaund Dodekapeptid, über Sephadex G 25 gereinigt. * Die Löslichkeit des Präparates war bei $p_{\mathrm{H}} 7,2$ zu gering, um in der zu diesem Test erforderlichen Konzentration gelöst zu werden.

B) Um auch die chemische Gleichheit der synthetischen A-Kette mit der aus natürlichem Schafinsulin $\mathrm{zu}$ beweisen, wurden beide unter gleichen Bedingungen partialhydrolysiert und mit der FingerprintTechnik verglichen.

Die Bedingungen der Hydrolyse entsprachen der Arbeitsweise von SANGER ${ }^{2}$ : Die Ketten wurden in der zum Cysteinsäure-Derivat oxydierten Form in 12-n. Salzsäure bei $37^{\circ}$ einen, zwei und drei Tage lang der Hydrolyse unterworfen. Zum Vergleich wurde daneben unter denselben Bedingungen die oxydierte A-Kette des Rinderinsulins hydrolysiert. Die Hydrolysate wurden zweidimensional [Butanol-2 /Eisessig/Wasser (75:13,5:11,5) und Phenol/Wasser $(80: 20)]$ chromatographiert. Die Peptidbruchstücke der synthetischen und der natürlichen A-Kette des Schafinsulins waren in der Anfärbung, Intensität und den Wanderungswegen völlig identisch (vgl. Abb. 3). Andererseits war der Unterschied zwischen den A-Ketten des Schaf- und des Rinderinsulins

11 J. Groen, C. E. Kamminge, A. F. Willebrands u. J. R. Blickman, J. clin. Invest. 31, 47 [1956]; J. Valdance-Owen u. B. HURLock, Lancet 1, 68 [1954].

12 H. Ditschuneit, J.-D. Faulhaber u. E. F. Pfeiffer, 8. Symp. Endokrinologie, München 1961, S. 387-392; Atompraxis 8, 172 [1962]. 


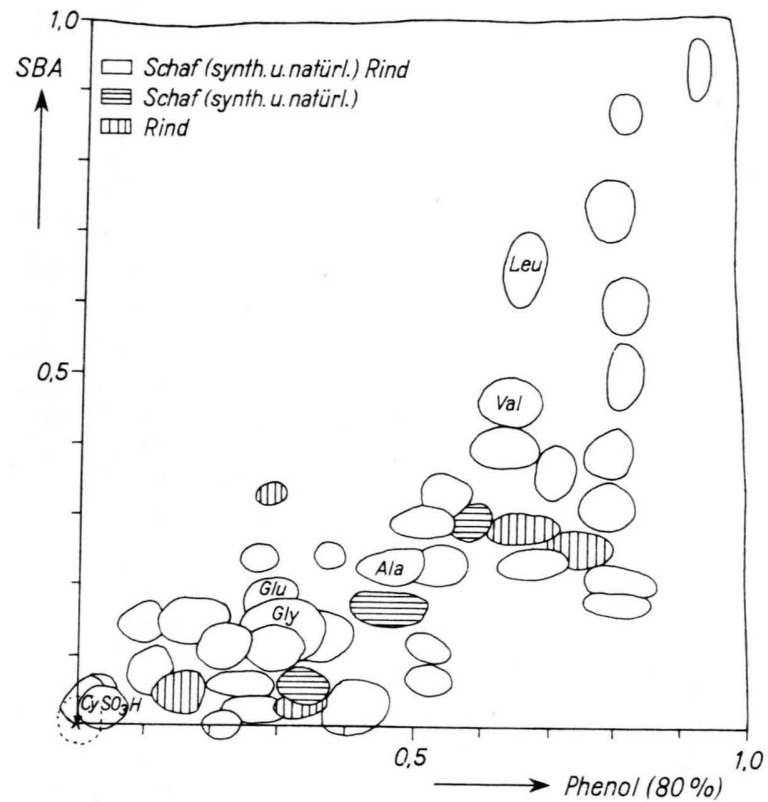

Abb. 3. Zweidimensionale Chromatographie (Papier Nr. 2043 b, Schleicher \& Schüll) von Partialhydrolysaten oxydierter Insulin-A-Ketten. Zwischen natürlicher und synthetischer A-Kette des Schafinsulins besteht kein Unterschied, Rindund Schaf-Kette unterscheiden sich dagegen durch acht Flecke.

deutlich zu erkennen. Als typisch für die A-Kette des Schafinsulins erscheinen drei Peptide, wogegen fünf nur bei der Partialhydrolyse des Rinderinsulins auftreten. Die beiden Hormone unterscheiden sich nur in der Position neun der A-Kette, das Rinderinsulin enthält dort einen Serinrest an Stelle des Glycinrestes (Schaf).

Die Ergebnisse der Abbauversuche und die biologische Aktivität des synthetischen und halbsynthetischen Insulins lassen den Schluß zu, daß die von Sanger ermittelte Formel für die A-Kette des Schafinsulins die richtige Sequenz darstellt und daß durch die Synthese ein Peptid gewonnen wurde, welches mit dem natürlichen übereinstimmt.

\section{Beschreibung der Versuche}

Alle Synthesen wurden mit L-Aminosäuren ausgeführt. Die Schmelzpunkte wurden mit dem Monoskop der Firma H. Bock, Bad Oeynhausen, bestimmt und mit Hilfe von Eichsubstanzen der Firma Reichert, Wien, korrigiert.

13 E. Fischer u. W. A. Jacobs, Ber. dtsch. chem. Ges. 39, 2942 [1906].

14 M. Bodanszky, Nature [London] 175, 685 [1955].

15 St. Gutmann u. R. A. Borssonnas, Helv. chim. Acta 43, 200 [1960].
Die $R_{f}$-Werte wurden in aufsteigender Technik dünnschichtchromatographisch auf mit Kieselgel G der Firma E. Merck, Darmstadt, beschichteten Platten bestimmt. Da die Schichtdicken nicht genau reproduziert werden konnten, sind alle $R_{f}$-Werte auf Leucin bezogen.

Laufmittel: Butanol-2/90-proz. Ameisensäure/Wasser $(75: 15: 10)$.

Abkürzungen: DMF = Dimethylformamid, TÄA = Triäthylamin, THF $=$ Tetrahydrofuran.

$$
\text { Synthesedes Dodek a peptids A } 10-21
$$

(Schema 2)

Ca r bobenzoxy-S - b enzyl-cystein ylserinmethylester (I)

$25 \mathrm{~g}(0,16 \mathrm{Mol}) \mathrm{HCl} \cdot \mathrm{H}-\mathrm{Ser}-\mathrm{OMe}{ }^{13}$ wurden in $150 \mathrm{ml} \mathrm{DMF}$ gelöst, mit $25 \mathrm{ml}(0,18 \mathrm{Mol}) \mathrm{TÄA}, 2,5 \mathrm{ml}$ Eisessig und einer Lösung von $75 \mathrm{~g}(0,16 \mathrm{Mol})$ Z-Cys (BZL) -ONP ${ }^{14}$ in $200 \mathrm{ml}$ DMF versetzt. Nach $48 \mathrm{Stdn}$. bei $0-5^{\circ}$ wurde der Ansatz unter Rühren in $3 l 0,1-n$. $\mathrm{KHCO}_{3}$-Lösung gegossen. Das ölige Produkt kristallisierte bald, wurde abgesaugt, mit Wasser gewaschen und aus Methanol und Äthylacetat umkristallisiert.

Ausbeute $47 \mathrm{~g}(65 \%)$, Schmp. $\left.111-112^{\circ},[\alpha]\right]_{\mathrm{D}}^{23}=$ $-29,8^{\circ}$ (c 2; DMF).

Lit. ${ }^{15}$ : Schmp. $111^{\circ},[\alpha]_{\mathrm{D}}^{22}=-31^{\circ}(c 1,8 ; \mathrm{DMF})$, $R_{\text {Leu }}=1,31$.

Di-(carbobenzoxy- $S$-benzyl-cysteinyl)serin-meth ylest er

Bei Verwendung von Äthylacetat oder Tetrahydrofuran als Lösungsmittel für die Umsetzung konnte durch fraktionierte Kristallisation aus Äthylacetat eine schwerer lösliche Komponente (Ausbeute bis zu 25\%) vom Schmp. $133-134^{\circ}$ und $[\alpha]_{\mathrm{D}}^{23}=-42,2^{\circ} \quad$ (c 2; DMF) isoliert werden. Nach der Elementaranalyse kann es sich um den diacylierten Serinmethylester handeln.

$\mathrm{C}_{40} \mathrm{H}_{43} \mathrm{O}_{9} \mathrm{~N}_{3} \mathrm{~S}_{2}(773,9) \quad R_{\text {Leu }}=1,48$.

Ber. C 62,08 H 5,60 N 5,43 S 8,29,

Gef. C 62,04 H 5,65 N 5,47 S 8,14.

C a r bobenzox y-valy $1-S-$ be nzy l-

cysteinyl-serinmethylester (II)

$5,0 \mathrm{~g}(0,011 \mathrm{Mol}) \mathrm{I}$ wurden in $25 \mathrm{ml}$ Trifluoressigsäure gelöst und trockenes $\mathrm{HBr}-\mathrm{Gas}$ durchgeleitet ${ }^{16}$. Nach $1^{1 / 2}$ Stdn. wurde die Lösung in $400 \mathrm{ml}$ absol. Äther eingerührt, der Niederschlag durch Dekantieren mit Äther gewaschen und aus Methanol/Äther umkristallisiert. Schmp. $140-142^{\circ},[\alpha]_{\mathrm{D}}^{25}=+4,0 \quad(c 2$; $\mathrm{MeOH})$.

4,50 g (0,011 Mol) $\mathrm{HBr} \cdot \mathrm{H}-\mathrm{Cys}(\mathrm{BZL})$-Ser-OMe wurden in $20 \mathrm{ml}$ DMF gelöst, mit $0,2 \mathrm{ml}$ Eisessig, $1,75 \mathrm{ml}$ TÄA und 4,26 $\mathrm{g}(0,0114 \mathrm{Mol}) \mathrm{Z}-\mathrm{Val}-\mathrm{ONP}{ }^{17}$ in $50 \mathrm{ml}$

16 St. Guttmann u. R. A. Boissonnas, Helv. chim. Acta 42, 1257 [1959].

17 B. Iselin, W. Rittel, P. Sieber u. R. Schwyzer, Helv. chim. Acta 40, 373 [1957]. 
DMF versetzt. Nach 5 Tagen bei $0^{\circ}$ wurde durch Einrühren in $1 l 0,1-n$. $\mathrm{KHCO}_{3}$-Lösung gefällt, abgesaugt, mit Wasser gewaschen und aus THF oder sek.-Butanol umkristallisiert.

Ausbeute $3,5 \mathrm{~g}$ (56\%), Schmp. 203-204, [a] ${ }_{\mathrm{D}}^{20}=$ $-28,0^{\circ}$ (c $\left.2 ; \mathrm{DMF}\right)$. DMF).

$$
\begin{aligned}
& \mathrm{C}_{27} \mathrm{H}_{35} \mathrm{O}_{7} \mathrm{~N}_{3} \mathrm{~S}(545,6) \quad R_{\text {Leu }}=0,95 \text {. } \\
& \text { Ber. C } 59,43 \text { H } 6,47 \text { N } 7,70 \text { S } 5,88 \text {, } \\
& \text { Gef. C } 59,67 \text { H } 6,35 \text { N 7,46 S 5,65. } \\
& \text { C a r bobenzoxy-valyl-S-benzyl- } \\
& \text { c y steinyl-serinhydrazid (III) }
\end{aligned}
$$

$10,0 \mathrm{~g}$ II wurden in einer Mischung aus $100 \mathrm{ml}$ DMF und $100 \mathrm{ml} \mathrm{Butanol-2}$ mit $5 \mathrm{ml}$ Hydrazinhydrat 1 Tag bei Raumtemperatur stehen gelassen. Das Hydrazid kristallisierte aus der Lösung, wurde abgesaugt und mit Methanol und Äther gewaschen. Ausbeute $9,4 \mathrm{~g}(94 \%)$, Schmp. $222-224^{\circ},[\alpha]_{\mathrm{D}}^{25}=-22,3^{\circ}(c$ $=1 ; \mathrm{DMF})$.

Lit. ${ }^{3}$ : Schmp. $230^{\circ},[\alpha]_{\mathrm{D}}^{29}=-19,6^{\circ}$ (c 0,58 ; DMF).

$$
\begin{aligned}
& \mathrm{C}_{26} \mathrm{H}_{35} \mathrm{~N}_{5} \mathrm{O}_{6} \mathrm{~S} \quad(545,6) \\
& \text { Ber. C } 57,23 \text { H } 6,47 \text { N } 12,84 \text { S 5,88, } \\
& \text { Gef. C } 56,99 \text { H 6,45 N 12,71 S 6,03. }
\end{aligned}
$$

Hydrazid-Stickstoff ${ }^{18}$, ber. 5,16 , gef. 5,37 .

Ca rbobenzoxy-valyl-S-benzylcysteinyl-seryl-leucyl-tyrosyl-glutaminyl-leucinmethylester

$$
\text { (IV) }
$$

\section{Bereitung des Azids}

$1,13 \mathrm{~g}(2,05 \mathrm{mMol})$ III wurden in $4 \mathrm{ml} 2-n . \mathrm{HCl}$, $10 \mathrm{ml}$ Eisessig und $10 \mathrm{ml}$ THF gelöst. Bei $-5^{\circ}$ wurden $147 \mathrm{mg}(2,13 \mathrm{mMol}) \mathrm{NaNO}_{2}$, gelöst in $1 \mathrm{ml} \mathrm{Was-}$ ser, zugesetzt. Nach 5 Min. bei $0^{\circ}$ wurde mit 20-proz. $\mathrm{K}_{2} \mathrm{CO}_{3}$-Lösung neutralisiert und das Azid durch zweimaliges Ausschütteln mit Äthylacetat extrahiert. Die Essigesterphase wurde einmal mit ges. NaCl-Lösung gewaschen und kurz über $\mathrm{Na}_{2} \mathrm{SO}_{4}$ getrocknet.

\section{Bereitung der Aminkomponente}

$1,50 \mathrm{~g}$ (2,19 mMol) Z-Leu-Tyr-Glu( $\left.\mathrm{NH}_{2}\right)$-Leu-OMe ${ }^{19}$

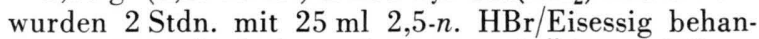
delt und das Hydrobromid mit $300 \mathrm{ml}$ Äther gefällt.

\section{Kondensation}

Das Tetrapeptidhydrobromid wurde in $10 \mathrm{ml}$ DMF gelöst, mit $0,4 \mathrm{ml}$ TÄA versetzt und mit der Azidlösung vereinigt. Die Reaktionsmischung blieb einen Tag bei $0^{\circ}$ und zwei weitere Tage bei Raumtemperatur stehen. Das ausgefallene Heptapeptid-Derivat wurde abgesaugt und mit Äthylacetat und Methanol gewaschen. Nach dem Umfällen aus DMF/Wasser betrug die Ausbeute

18 H. Medzihradszky-Schweiger, Acta chim. Acad. Sci. hung. 34, 213 [1962].
$1,15 \mathrm{~g}(53 \%)$, Schmp. $247-248^{\circ},[\alpha]_{\mathrm{D}}^{25}=-36,4^{\circ}$ (c $1 ; \mathrm{DMF})$.

$$
\begin{aligned}
& \mathrm{C}_{53} \mathrm{H}_{74} \mathrm{~N}_{8} \mathrm{O}_{13} \mathrm{~S} \quad(1063,3) \quad R_{\text {Leu }}=1,40 \text {. } \\
& \text { Ber. C. } 59,88 \text { H } 7,02 \text { N } 10,54 \text { S } 3,03 \text {, } \\
& \text { Gef. C } 59,75 \text { H } 6,97 \text { N 10,44 S } 3,23 \text {. }
\end{aligned}
$$

Carbobenzoxy-valyl-S-benzylcysteinyl-seryl-leucyl-tyrosyl-glutaminyl-le u cin hy d razid (V)

$1,85 \mathrm{~g}(1,73 \mathrm{mMol})$ IV wurden in $20 \mathrm{ml}$ DMF gelöst und nach Zusatz von $0,85 \mathrm{ml}$ Hydrazinhydrat fünf Tage bei Raumtemperatur stehen gelassen. Die gelierte Masse wurde mit $20 \mathrm{ml}$ Dioxan und $20 \mathrm{ml}$ Wasser verrührt. Das Gel wurde abgesaugt und mit Wasser und Methanol gewaschen. Dann wurde mit Methanol aufgekocht und wieder abgesaugt.

Ausbeute $1,57 \mathrm{~g}$ (85\%), Schmp. 248-250 ${ }^{\circ}$ Zersetzung. [a] ${ }_{\mathrm{D}}^{25}=-38,4^{\circ}$ ( $c 1$; Ameisensäure).

$\mathrm{C}_{52} \mathrm{H}_{74} \mathrm{~N}_{10} \mathrm{O}_{12} \mathrm{~S} \quad(1063,3)$

Hydrazid-Stickstoff ${ }^{18}$ : Ber. 2,63, gef. 2,64.

C a r bobenzoxy-valyl-S-benzylcysteinyl-seryl-leucyl-tyrosyl-glutaminyl-leucyl-glut a my l-a sparaginyl-

tyrosyl-S-benzyl-cysteinyl-a sparagin (VI)

\section{Bereitung des Azids}

$1,06 \mathrm{~g}$ ( $1 \mathrm{mMol}) \mathrm{V}$ wurden in $15 \mathrm{ml}$ Eisessig und 3 ml 1-n. HCl gelöst. Nach Kühlung auf $-2^{\circ}$ wurde eine Lösung von $69 \mathrm{mg}$ (1 mMol) Natriumnitrit in $1 \mathrm{ml}$ Wasser zugefügt, worauf das Azid bald ausfiel. Es wurde ohne Isolierung nach 10 Min. in Äthylacetat von $0^{\circ}$ suspendiert und die Suspension mit $0,1-n$. $\mathrm{KHCO}_{3}$ und NaCl-Lösung gewaschen. Auf Zusatz von $100 \mathrm{ml}$ DMF ging das Azid in Lösung. Der Essigester wurde im Vakuum abgedampft. Bei allen diesen Operationen stieg die Temperatur nicht über $0^{\circ}$.

\section{Bereitung der Aminkomponente}

$0,92 \mathrm{~g}(1 \mathrm{mMol}) \quad \mathrm{Z}-\mathrm{Glu}\left(\mathrm{OBu}^{\mathrm{t}}\right)-\mathrm{Asp}\left(\mathrm{NH}_{2}\right)-\mathrm{Tyr}-\mathrm{Cys}$ (BZL)-Asp $\left(\mathrm{NH}_{2}\right)^{7}$ wurden eine Stde. mit $20 \mathrm{ml}$ 2,5-n. $\mathrm{HBr} /$ Eisessig behandelt und die Lösung dann in $600 \mathrm{ml}$ absol. Äther eingerührt. Der Niederschlag wurde abgesaugt, mit Äther gewaschen und im Vakuum über $\mathrm{KOH} / \mathrm{P}_{2} \mathrm{O}_{5}$ getrocknet. Das so gewonnene Hydrobromid

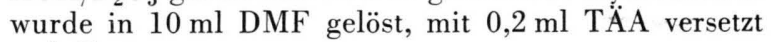
und filtriert.

\section{Kondensation}

Die Azidlösung wurde bei $0^{\circ}$ mit der Lösung der Aminkomponente vereinigt und die Mischung 3 Tage bei $0^{\circ}$ stehen gelassen. Durch Einrühren in $1 l$ Eiswasser, dem die etwa äquivalente Menge $\mathrm{HCl}$ zugesetzt worden war, wurde die Peptidsäure gefällt. Nach dem Einstellen des $p_{H^{-}}$Wertes auf $2-3$ blieb die Fällung

19 P. G. Katsoyannis, K. Suzuki u. A. Tometsko, J. Amer. chem. Soc. 85, 1139 [1963]. 
noch 1 Stde. stehen, dann wurde abgesaugt, gut mit Wasser gewaschen und getrocknet. Rohausbeute $1,32 \mathrm{~g}$ $(74 \%)$.

Das Rohprodukt ließ sich durch zweimaliges Auskochen mit Methanol auf einen konstanten Schmelzpunkt bringen und von im Dünnschichtchromatogramm sichtbaren Verunreinigungen befreien. Ausbeute $0,80 \mathrm{~g}$ $(45 \%) ; R_{\text {Leu }} 1,38$.

Schmp. $257-259^{\circ},[\alpha]{ }_{\mathrm{D}}^{25}=-37,5^{\circ}$ (c 1; Ameisensäure).

$$
\begin{aligned}
& \mathrm{C}_{84} \mathrm{H}_{111} \mathrm{~N}_{15} \mathrm{O}_{23} \mathrm{~S} \quad(1763,0) \\
& \text { Ber. C } 57,22 \text { H 6,35 N 11,92 S 3,64, } \\
& \text { Gef. C 56,73 H 6,21 N 12,27 S 3,53. }
\end{aligned}
$$

Synthese des Heneikosipeptids A $1-21$ (Schema 1)

Carbobenzoxy-glycyl-isoleucyl-valylglut a myl-glutaminyl- $S$ - benzyl-

cysteinyl- $S$ - benzyl-cysteinyl-alanylglycyl-valyl-S-benzyl-cysteinylseryl-leucyl-tyrosyl-glutaminyl-

leucyl-glutamyl-a sparaginyl-t y ros yl$S$-benzyl-cysteinyl-asparagin (VII)

\section{Bereitung der Aminkomponente}

$300 \mathrm{mg}(0,17 \mathrm{mMol})$ VI wurden in $8 \mathrm{ml}$ Trifluoressigsäure gelöst und während $30 \mathrm{Min}$. trockenes $\mathrm{HBr}$ durchgeleitet ${ }^{13}$. Dann wurde im Vakuum eingedampft und das zurückbleibende Öl mit Benzol verrieben. Nach Abdampfen des Benzols wurde mit absol. Äther verrührt, das feste Produkt abgesaugt und mit Äther gewaschen. Das Hydrobromid wurde in 2,5 ml Hexamethylphosphorsäuretriamid gelöst und mit $0,09 \mathrm{ml}$ (0,65 mMol) TÄA versetzt.

\section{Bereitung des gemischten Anhydrids}

$215 \mathrm{mg} \quad(0,17 \mathrm{mMol}) \quad$ Z-Gly-Ile-Val-Glu $\left(\mathrm{OBu}^{\mathrm{t}}\right)-\mathrm{Glu}$ $\left(\mathrm{NH}_{2}\right)$-Cys (BZL)-Cys (BZL)-Ala-Gly ${ }^{6}$ (vorher 1 Stde. bei $95^{\circ}$ im Vakuum über $\mathrm{P}_{2} \mathrm{O}_{5}$ getrocknet) wurden in $1,7 \mathrm{ml}$ absol. Hexamethylphosphorsäuretriamid gelöst und mit $0,025 \mathrm{ml}(0,18 \mathrm{mMol})$ TÄA versetzt. Nach Kühlung auf $-30^{\circ}$ wurden $0,0164 \mathrm{ml}(0,17 \mathrm{mMol})$ Chlorameisensäureäthylester zugegeben und 15 Min. bei $-20^{\circ}$ gerührt.

\section{Kondensation}

$\mathrm{Zu}$ der $-20^{\circ}$ kalten Lösung des gemischten Anhydrids wurde die ebenfalls gekühlte Lösung der Aminokomponente gegeben. Das Gemisch wurde bei $-10^{\circ}$ noch $3 \mathrm{Stdn}$. gerührt und anschließend 24 Stdn. bei $0^{\circ}$ aufbewahrt. Durch Einrühren in $200 \mathrm{ml}$ mit $\mathrm{NaCl}$ gesättigtes Wasser wurde das Peptid ausgefällt. Nach 2 Stdn. wurde der feinpulvrige Niederschlag abgesaugt und gründlich mit Wasser gewaschen. Das Produkt wurde noch feucht in $15 \mathrm{ml} \mathrm{DMF}$ und $3 \mathrm{ml} \mathrm{Me-}$ thanol gelöst und durch Einrühren in $300 \mathrm{ml}$ Äther wieder ausgefällt. Nach dem Trocknen betrug die Ausbeute $480 \mathrm{mg}$.

\section{Acidolyse des Glu'- $\gamma$-tert.-Butylesters}

$480 \mathrm{mg}$ des Rohproduktes wurden in $6 \mathrm{ml}$ Trifluoressigsäure gelöst. Nach 1-stdg. Stehen wurde im Vakuum eingedampft, der Rückstand mit Äther verrieben und abgesaugt. Ausbeute $444 \mathrm{mg}$ (94\%), Schmp. $>300^{\circ}$ Zersetzung. [ $\left.\alpha\right]_{\mathrm{D}}^{25}=-48^{\circ}(c 1 ; \mathrm{HCOOH})$.

Beim Auskochen mit Methanol gingen 15\% der Substanz in Lösung. Der Rückstand hatte nach dem Trocknen einen Drehwert von $[\alpha]_{\mathrm{D}}^{25}=-50^{\circ}(c 1 ; \mathrm{HCOOH})$.

\section{Gelfiltration}

$50 \mathrm{mg}$ des teilgeschützten Heneikosipeptids und $60 \mathrm{mg}$ TRIS-Puffer (Tris-hydroxymethyl-aminomethan) wurden in $1 \mathrm{ml}$ Wasser gelöst, mit weiteren $4 \mathrm{ml}$ Wasser verdünnt und mit $0,1-n . \mathrm{HNO}_{3}$ auf $p_{\mathrm{H}} 8,5-8,7$ gebracht. Diese Lösung wurde auf eine Säule (Länge $670 \mathrm{~mm}$, Durchmesser $28 \mathrm{~mm}$, Füllung Sephadex G 25 fine, eingeschlämmt mit $0,1 \mathrm{~m}$ TRIS-Puffer vom $p_{\mathrm{H}}$ $8,5-8,7)$ gegeben und mit $0,1 \mathrm{~m}$ TRIS-Puffer vom $p_{\mathrm{H}} \quad 8,5-8,7$ eluiert. Die UV-Absorptionskurve des Eluats zeigte zwei Peaks. Die entsprechenden Fraktionen wurden aufgefangen, mit verd. $\mathrm{HNO}_{3}$ auf $p_{\mathrm{H}} 3$ angesäuert, wobei die Peptide ausflockten. Die so erhaltenen Suspensionen wurden in Viskingschlauch 18/32 drei Tage gegen Wasser dialysiert und gefriergetrocknet. Die erste Fraktion ergab $43 \mathrm{mg}$ und erwies sich als A-Ketten-Derivat. Zersetzung über $300^{\circ}$, kein Schmelzpunkt. [a] ${ }_{\mathrm{D}}^{25}=-51^{\circ}($ c $1 ; \mathrm{HCOOH})$.

$\mathrm{C}_{132} \mathrm{H}_{179} \mathrm{~N}_{25} \mathrm{O}_{35} \mathrm{~S}_{4}, 5 \mathrm{H}_{2} \mathrm{O} \quad(2894,3)$

Ber. C 54,77 H 6,58 N 12,10 S 4,43 O 22,11,

Gef. C 53,95 H 6,51 N 12,06 S 5,48 O 21,95.

\section{Quant. Aminosäureanalyse und Amidbestimmung} vgl. S. 656

Die zweite Fraktion schmolz bei $250-253^{\circ}$. Im zweidimensionalen Papierchromatogramm fanden sich alle Aminosäuren des Nonapeptids A $1-9$ sowie ein weiterer Fleck, dessen $R_{f}$-Werte mit denen des Dipeptids Ile-Val übereinstimmten. Eine Probe Z-Gly-Ile-Val$\mathrm{Glu}\left(\mathrm{OBu}^{\mathrm{t}}\right)$ - Glu $\left(\mathrm{NH}_{2}\right)$ - Cys (BZL) - Cys (BZL) - Ala - Gly hatte nach Spaltung der tert.-Butylestergruppe des $\mathrm{Glu}^{4}$-Restes einen Schmelzpunkt von $253-255^{\circ}$.

\section{Kombination der synthetischen A-Kette mit natürlicher Insulin-B-Kette}

$29 \mathrm{mg}$ des gereinigten A-Kettenderivates VII und $43 \mathrm{mg}$ B-Kette-7,19-bis-thiosulfat ${ }^{20}$ wurden vermischt und im Hochvakuum $5 \mathrm{Stdn}$. bei $55^{\circ}$ über Phosphorpentoxid getrocknet. Die Reaktion mit Natrium in flüssigem Ammoniak und Weiterverarbeitung erfolgten in der von $Z_{\mathrm{AHN}}$ et al. ${ }^{9}$ beschriebenen Weise bei $p_{\mathrm{H}} 9,0$. Ausbeute: $30 \mathrm{mg}$ (69\% d. Th.).

$$
\text { S e quenzverglei c he }
$$

\section{a) Deblockierung und Oxydation}

$33 \mathrm{mg}$ der gereinigten teilgeschützten A-Kette des Schafinsulins (VII) wurden drei Stdn. bei $50^{\circ}$ im Va-

20 J. L. BAiley, Biochem. J. 67, 21 P [1957]. 
kuum über $\mathrm{P}_{2} \mathrm{O}_{5}$ getrocknet und in $100 \mathrm{ml}$ über $\mathrm{Na}$ trium dest. flüssigem Ammoniak gelöst. Die Schutzgruppen wurden durch Reduktion mit Natrium (portionsweise Zugabe bis zur bleibenden Blaufärbung) innerhalb von $40 \mathrm{Min}$. abgespalten. Die restliche, nach 10 Min. noch verbliebene Blaufärbung wurde durch tropfenweise Zugabe von 0,5 ml Eisessig entfernt. Nach Abdampfen des Ammoniaks wurde der weiße Rückstand in $50 \mathrm{ml}$ Wasser aufgenommen, mit Citronensäure-Lösung (10\%) auf $p_{\mathrm{H}} 5$ gebracht und $22 \mathrm{Stdn}$. im Visking-Schlauch 18/32 gegen Wasser dialysiert. Eine Prüfung auf Thiolgruppen mit Nitroprussidnatrium fiel schon vor der Dialyse negativ aus. Nach der Gefriertrocknung wurden $23 \mathrm{mg}=86 \%$ als weiße, pulvrige Substanz erhalten.

Die weitere Oxydation zum Cysteinsäurepeptid erfolgte mit Perameisensäure ${ }^{21}: 4 \mathrm{ml}$ Ameisensäure $(99 \%)$ und $1 \mathrm{ml} \mathrm{H}_{2} \mathrm{O}_{2}(30 \%)$ wurden nach $15 \mathrm{Min}$. Reaktionsdauer bei Raumtemperatur auf $0^{\circ}$ gekühlt und $20 \mathrm{mg}$ des Peptids (in der Disulfidform) darin gelöst. Nach 30 Min. Oxydation bei $0^{\circ 22}$ wurde die überschüssige Perameisensäure durch Zugabe von $5 \mathrm{ml}$ Wasser zerstört. Anschließend wurde im Vakuum abgedampft, wobei zweimal nach Einengung auf die Hälfte $5 \mathrm{ml}$ Wasser zugesetzt wurden. Die wäßrige Lösung ließ sich durch Gefriertrocknung in ein weißes, lockeres Pulver überführen.

\section{b) Partialhydrolyse}

Je $2,5 \mathrm{mg} \quad(0,001 \mathrm{mMol})$ der oxydierten synthetischen und natürlichen A-Kette des Schafinsulins sowie je 2,5 mg der oxydierten A-Kette des natürlichen Rinderinsulins wurden zusammen mit je $1 \mathrm{ml} 12-n . \mathrm{HCl}$ in Hydrolyseröhrchen eingeschmolzen und 24, 48 und $72 \mathrm{Stdn}$. bei $37^{\circ}$ hydrolysiert. Die Hydrolysate wurden in 10-ml-Bechergläser übergeführt und sofort im Vakuum bei Raumtemperatur über $\mathrm{P}_{2} \mathrm{O}_{5}$ und $\mathrm{KOH}$ ge-

21 G. Toennies u. R. P. Homiller, J. Amer. chem. Soc. 64, 3054 [1942]. trocknet, der Rückstand in einigen Tropfen Wasser gelöst und zur Entfernung der überschüssigen Salzsäure nochmals im Vakuum getrocknet.

Zum Chromatographieren wurde in $0,2 \mathrm{ml}$ Wasser gelöst und davon $0,1 \mathrm{ml}$ so auf den Startpunkt aufgetragen, daß der Auftragfleck nicht mehr als $10 \mathrm{~mm}$ Durchmesser hatte (nach je 0,02 ml Auftrag getrocknet). Chromatographiert wurde zuerst in Butanol-2/ Ameisensäure/Wasser und dann in Phenol (80\%). Entwickelt wurde mit Ninhydrinlösung. Die Chromatogramme wurden nach dem Sprühen an der Luft getrocknet und danach 1 Min. im Trockenschrank auf $110^{\circ}$ erhitzt. Die Farbe und Lage der gebildeten Flekken wurde nach einer Stde. notiert (vgl. Abb. 3).

Wir danken den Herren Dr. J. Meienhofer, Dr. E. Schnabel und Dr. O. Brinkhoff für wertvolle Ratschläge, den Herren Dr. Kl. Ziegler, Dr. H. Klostermeyer und Dr. J. Meienhofer für analytische Untersuchungen sowie den Herren Prof. Dr. E. F. Pfeiffer und Dr. H. Ditschuneit, Frankfurt, Dr. C. Gloxhuber, Farbenfabriken Bayer, Leverkusen, und Prof. H. CLAUsER, Paris, für biologische Teste. Weiter danken wir dem Bundeswirtschaftsministerium, Bonn, (Forschungsvorhaben 822), der A r b e its gemeinschaft industrieller Forschungsvereinigungen, Köln, dem Forschungs kuratorium Gesamttextil, Frankfurt, dem Landesamt für Forschung beim Ministerpräsidenten des Landes NordrheinWestfalen, Düsseldorf, und der Deut s chen Forschungsgemein schaft, Bad Godesberg, für finanzielle Unterstützung der vorliegenden Arbeit. Dem Verband der chemis chen Industrie, Düsseldorf, und seinen Mitgliedsfirmen danken wir für die Überlassung von Chemikalien im Rahmen von Hochschullieferungen.

22 E. O. P. Thомpson, Biochim. biophysica Acta [Amsterdam] 15, 440 [1954]. 